\title{
Rotons in a Hybrid Bose-Fermi System
}

\author{
Ivan A. Shelykh \\ Science Institute, University of Iceland, Dunhagi-3, IS-107, Reykjavik, Iceland and International Institute for Physics, \\ UFRN-Universidade Federal do Rio Grande do Norte, Campus Universitario Lagoa Nova, CEP: 59078-970, Natal- RN, Brazil \\ Thomas Taylor and Alexey V. Kavokin \\ School of Physics and Astronomy, University of Southampton, Highfield Southampton, SO171BJ, United Kingtom
}

(Received 17 June 2010; revised manuscript received 10 August 2010; published 28 September 2010)

\begin{abstract}
We calculate the spectrum of elementary excitations in a two-dimensional exciton condensate in the vicinity of a two-dimensional electron gas. We show that attraction of excitons due to their scattering with free electrons may lead to formation of a roton minimum. The energy of this minimum may go below the ground state energy which manifests breaking of the superfluidity. The Berezinsky-Kosterlitz-Thouless phase transition temperature decreases due to the exciton-exciton attraction mediated by electrons.
\end{abstract}

DOI: 10.1103/PhysRevLett.105.140402

PACS numbers: 05.30.Jp, 05.30.Fk, 73.22.Lp, 78.67.Pt

In this Letter we consider a hybrid Bose-Fermi system consisting of a spatially separated but interacting BoseEinstein condensate (BEC) and Fermi sea. Such systems have been discussed in relation to cold atom gases and shown to be extremely rich in fundamental effects including the BCS-BEC crossover [1], Tonks- Girardeau gases [2], Cooper pairing, and superconductivity [3]. Hybrid systems remain poorly studied experimentally as the realization of spatially separated but interacting Fermi-sea and Bose-Einstein condensate of cold atoms is a nontrivial task. Recently, the potential of semiconductor coupled quantum wells for the realization of BEC of cold excitons has been revealed [4]. Spatially indirect excitons were widely studied both experimentally and theoretically in recent years (see Ref. [5] for a review). They may strongly interact with remote free electrons due to their dipole moments. This is why the realization of a hybrid Bose-Fermi system in a semiconductor structure containing $n$-doped quantum wells (QWs), where the two-dimensional electron gas (2DEG) is created, and undoped coupled QWs, where the exciton BEC is induced, seems realistic. Recently, a proposal has been made for the realization of excitonmediated superconductivity in microcavity structures [6].

Here we study the effect of interaction of a BEC of spatially indirect excitons with a 2DEG on the energy spectrum of excitations of the exciton BEC. It is well established that in the absence of free electrons, the Bogoliubov-like spectrum showing linear dispersion near the ground state and parabolic dispersion at larger wave vectors is formed due to exciton-exciton repulsion. We show that due to their scattering with free electrons, excitons with nonzero momenta attract each other, which leads to the formation of a rotonlike minimum [7] in the exciton dispersion. The depth of the minimum increases with the increase of the exciton concentration, and consequently the roton gap decreases. In this regime, the critical temperature of the Berezinsky-Kosterlitz-Thouless (BKT) transition for excitons [8] decreases. Eventually, the roton energy goes below the ground state energy, and the roton gap collapses, leading to the collapse of the exciton BEC.

Consider a system of three parallel semiconductor quantum wells (QWs), one of which contains a free electron (or hole) gas, and two others containing a BEC of spatially indirect excitons. The effective interaction between electrons and excitons can be represented in diagrammatic form as is shown in Fig. 1. This figure shows the random phase approximation (RPA) diagrams for the electronic system and for the virtual excitations of the exciton BEC. The first diagram represents the direct interaction of two excitons and all subsequent ones correspond to the processes involving the virtual excitation of the Fermi sea or/ and excitonic BEC at intermediate stages. For example, the second diagram corresponds to the process of exciton interaction with the electron Fermi sea creating a virtual electron-hole pair in it, which afterwards disappears due to its interaction with another exciton. Diagram 3 corresponds to the process of exciton interaction with the condensate creating its virtual excitation, which then disappears due to the interaction with another exciton. Other diagrams correspond to higher-order processes where several virtual excitations are created at intermediate stages.

Using the standard rules for the evaluation of Feynman diagrams [9], one can obtain the matrix elements of the effective screened interaction:

$$
\begin{aligned}
V_{12}^{\text {eff }}= & V_{12}\left[1+\left(V_{11} \Pi_{1}+V_{22} \Pi_{2}\right)+\left(V_{11}^{2} \Pi_{1}^{2}+V_{22}^{2} \Pi_{2}^{2}\right.\right. \\
+ & \left.\left.V_{11} V_{22} \Pi_{1} \Pi_{2}+V_{12}^{2} \Pi_{1} \Pi_{2}\right)+\ldots\right], \\
V_{11}^{\text {eff }}= & V_{11}+\left(V_{11}^{2} \Pi_{1}+V_{12}^{2} \Pi_{2}\right)+\left(V_{11}^{3} \Pi_{1}^{2}\right. \\
& \left.+2 V_{11} V_{12}^{2} \Pi_{1} \Pi_{2}+V_{12}^{2} V_{22} \Pi_{2}^{2}\right)+\ldots \\
V_{22}^{\text {eff }}= & V_{22}+\left(V_{12}^{2} \Pi_{1}+V_{22}^{2} \Pi_{2}\right)+\left(V_{22}^{3} \Pi_{2}^{2}\right. \\
& \left.+2 V_{22} V_{12}^{2} \Pi_{1} \Pi_{2}+V_{12}^{2} V_{11} \Pi_{1}^{2}\right)+\ldots
\end{aligned}
$$




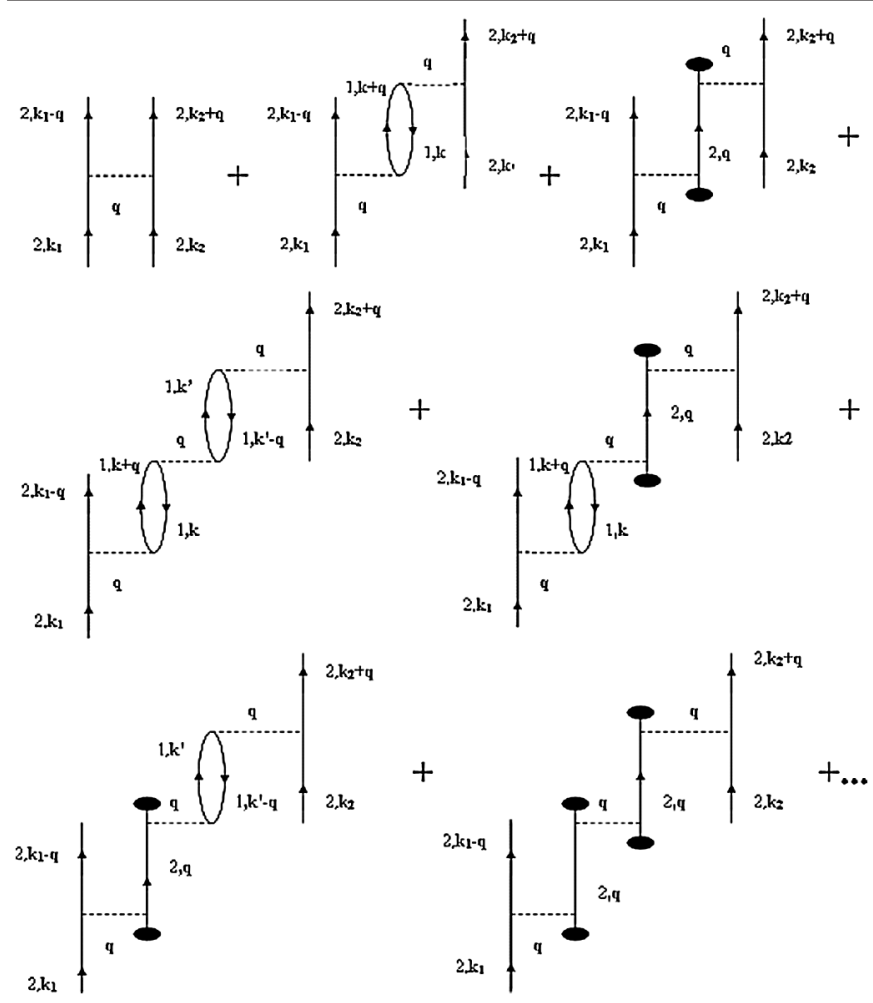

FIG. 1. RPA diagrammatic representation of the screened interaction between excitons and electrons. Index 1 corresponds to electrons, index 2 to excitons. Black dots correspond to the exciton BEC.

where $V_{11}(\mathbf{q})$ is the matrix element of the unscreened interaction between electrons, $V_{22}(\mathbf{q})$ is the matrix element of the unscreened interaction between excitons, $V_{12}(\mathbf{q})$ is the matrix element of the unscreened interaction between electrons and excitons. $\Pi_{j}=\Pi_{j}(\mathbf{q}, \omega)$ are polarization operators. For the electron system one can obtain [10]

$$
\Pi_{1}(\mathbf{q}, \omega)=\sum_{\mathbf{k}} \frac{f_{\mathbf{k}-\mathbf{q}}-f_{\mathbf{k}}}{\hbar\left(\omega+i \delta+E_{\mathbf{k}-\mathbf{q}}^{\mathrm{el}}-E_{\mathbf{k}}^{\mathrm{el}}\right)},
$$

and for the condensate [9]

$$
\Pi_{2}(\mathbf{q}, \omega)=N_{0} G_{0}^{\mathrm{ex}}(\mathbf{q}, \omega)=\frac{2 N_{0} E_{\mathbf{q}}^{\mathrm{ex}}}{(\hbar \omega)^{2}-\left(E_{\mathbf{q}}^{\mathrm{ex}}\right)^{2}}
$$

where $N_{0}$ is the occupation number of the condensate, $E_{\mathbf{q}}^{\text {ex }}$ and $E_{\mathbf{q}}^{\mathrm{el}}$ are dispersions of the bare excitons and electrons, respectively (parabolic, in the effective mass approximation), $f_{\mathbf{q}}$ is the Fermi distribution. The electron-electron interaction is described by a standard 2D Coulomb potential,

$$
V_{11}=\frac{e^{2}}{2 \epsilon_{0} \epsilon A} \frac{1}{q},
$$

with $\epsilon$ being a dielectric constant of the media and $A$ being the sample area. The matrix element of exciton-exciton interaction $V_{22}$ can be estimated as [11,12]

$$
V_{22}(q) \approx \frac{6 E_{B} a_{B}^{2}}{A}
$$

with $E_{B}$ and $a_{B}$ being the exciton binding energy and Bohr radius, respectively. We neglect the $q$-dependent contribution due to dipole-dipole interaction of excitons, which is essential for the problem of quantum diffusion of indirect excitons [13] but is exactly 0 for $q=0$ and small compared to exciton-electron interaction for small $q$ in the case of a uniform exciton BEC, which we consider here. The expression for the matrix element of electron-exciton interaction reads (the details of calculation can be found in, e.g., Ref. [14]):

$$
\begin{aligned}
V_{12}(q)= & \frac{e d e^{-q L}}{2 \epsilon_{0} \epsilon A}\left\{\frac{\beta_{e}}{\left[1+\left(\frac{\beta_{e} q a_{B}}{2}\right)^{2}\right]^{3 / 2}}+\frac{\beta_{h}}{\left[1+\left(\frac{\beta_{h} q a_{B}}{2}\right)^{2}\right]^{3 / 2}}\right\} \\
& +\frac{e^{2} e^{-q L}}{2 \epsilon_{0} \epsilon q A}\left\{\frac{1}{\left[1+\left(\frac{\beta_{e} q a_{B}}{2}\right)^{2}\right]^{3 / 2}}-\frac{1}{\left[1+\left(\frac{\beta_{h} q a_{B}}{2}\right)^{2}\right]^{3 / 2}}\right\},
\end{aligned}
$$

where $\beta_{e, h}=m_{e, h} /\left(m_{e}+m_{h}\right)$ with $m_{e, h}$ being the effective masses of electron and hole, $d$ being the dipole moment of the exciton in normal to the QW plane direction. In order to interact efficiently with electrons, the excitons must have a significant dipole moment $\left(d \sim a_{B}\right)$ which is readily achieved in biased coupled QW structures where one of the wells confines electrons and another one confines holes [5].

The formula for the effective interaction can be rewritten in a compact matrix form

$$
\mathbf{V}^{\text {eff }}=\mathbf{V} \cdot(1-\Pi \mathbf{V})^{-1},
$$

where $\mathbf{V}^{\text {eff }}, \mathbf{V}$, and $\Pi$ are $2 \times 2$ matrices with matrix elements given above. The polarization matrix $\Pi$ is diagonal, with the diagonal elements given by expressions (4) and (5).

Expressions (9) fully determine all renormalized interactions in the electron-exciton system and describe simultaneously the screening effects, the Bogoliubov renormalization of the dispersion of the excitations of the condensate, the bogolon-mediated pairing of electrons responsible for exciton-mediated superconductivity [6], and effective attraction between excitons due to their scattering with electrons. The matrix element of effective exciton-exciton interaction taking into account all these effects reads

$$
\begin{aligned}
& V_{\mathrm{ex}-\mathrm{ex}}^{\mathrm{eff}}(q, \omega) \\
& =\frac{V_{22}+\frac{V_{12}^{2}(q) \Pi_{1}(q, \omega)}{1-V_{11}(q) \Pi_{1}(q, \omega)}}{(\hbar \omega)^{2}-\left[E^{\mathrm{ex}}(q)\right]^{2}-2 N_{0}\left[V_{22}+\frac{V_{12}^{2}(q) \Pi_{1}(q, \omega)}{1-V_{11}(q) \Pi_{1}(q, \omega)}\right] E^{\mathrm{ex}}(q)} .
\end{aligned}
$$

The first term in the numerator corresponds to the direct repulsive exciton-exciton interaction, while the second 
term describes the effective interaction between excitons due to the virtual excitations in the electronic system. If one neglects electron-electron interactions and polarizability assuming $V_{11}(q)=0, \Pi(q, \omega)=0$, for the effective interactions one recovers the result of Refs. [3,15].

The poles of the effective potential determine the dispersions of the collective modes of the system, given by the equation

$$
(\hbar \omega)^{2}=\left[E^{\mathrm{ex}}(q)\right]^{2}+2 N_{0} \tilde{V}_{22}(q) E^{\mathrm{ex}}(q),
$$

where

$$
\tilde{V}_{22}(q)=V_{22}+V_{12}^{2}(q) \Pi_{1}(q, \omega) /\left[1-V_{11}(q) \Pi_{1}(q, \omega)\right] .
$$

If the excitonic and electronic systems are uncoupled, $V_{12}=0$, two independent collective modes coexist: the Bogoliubov excitations of the condensate (bogolons) whose dispersion is given by $\hbar \omega_{B}(q)=$ $\sqrt{E^{\mathrm{ex}}(q)\left[E^{\mathrm{ex}}(q)+2 V_{22} N_{0}\right]}$, and the plasmon mode whose dispersion can be obtained from the transcendental equation $1-V_{11}(q) \Pi_{1}(q, \omega)=0$. On the other hand, if $V_{12} \neq$ 0 and $\Pi_{1} \neq 0$ dispersions of bogolons and plasmonic excitations are coupled.

In this regime, the roton minimum may appear in the energy spectrum of the exciton condensate. One can see that $\tilde{V}_{22}(0)=V_{22}$ as $V_{11}(0)=\infty$. Assuming $\omega \approx 0$ and using for $\Pi_{1}$ the static approximation, $\Pi_{1} \approx A \frac{m_{\mathrm{el}}}{\pi \hbar^{2}} \times$ $\left(e^{-\pi \hbar^{2} n_{\mathrm{el}} / k_{B} T m_{\mathrm{el}}}-1\right)<0$ where $m_{\mathrm{el}}$ and $n_{\mathrm{el}}$ are the effective masses and 2D concentration of the electrons, respectively [10], one can easily see that the second term in the right part of Eq. (12) is negative, and $\tilde{V}_{22}$ is a decreasing function of $q$, which is responsible for the appearance of the roton minimum.

The position and depth of this minimum are dependent on the strength of the exciton-electron interaction as Fig. 2 shows.

The minimum becomes deeper if the distance between the QW containing the electron gas and the exciton BEC decreases. It also deepens with increase of the exciton concentration in the condensate. At some critical concentration the energy of the roton minimum equals the energy of the condensate at $q=0$, so that the roton gap collapses. This manifests itself as a phase transition in the system: beyond this point the exciton BEC becomes unstable and eventually collapses due to uncontrollable escape of excitons towards the roton minimum. The physical reason for suppression of the exciton BEC is the attraction between excitons induced by their interaction with free electrons.

Figure 3 shows the phase diagram of the transition between the exciton BEC and classical condensation. One can see that the collapse of the BEC may be achieved at excitons concentrations much below the Mott density provided that the distance between excitons and electrons is small enough.

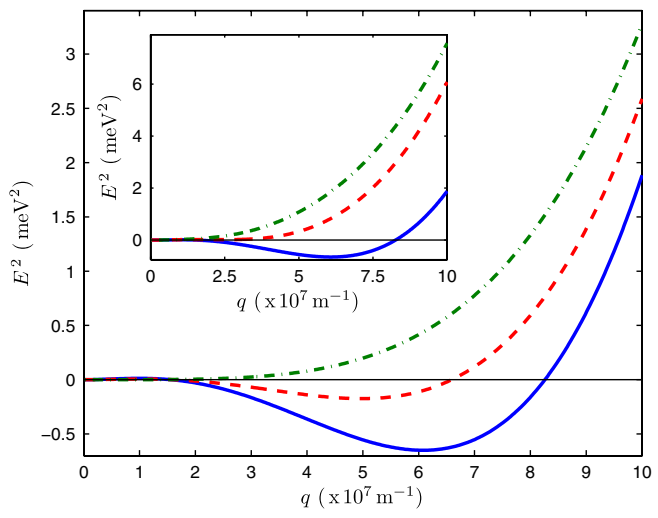

FIG. 2 (color online). Dispersion of the elementary excitations of the condensate showing roton minimum. Plotted for parameters of a coupled QW structure studied in Ref. [4] assuming that a third $n$-doped QW is grown at a distance $L$ from the coupled QW structure, the dipole separation is $l=12 \mathrm{~nm}$ and $n_{\mathrm{el}}=4 \times$ $10^{12} \mathrm{~cm}^{-2}$. In the main plot $L=12 \mathrm{~nm}$ and $n_{s}$ varies as $1,0.5$, $0.01 \times 10^{11} \mathrm{~cm}^{-2}$ (solid blue, dashed red, and dot-dashed green, respectively). In the inset $n_{s}=1 \times 10^{11} \mathrm{~cm}^{-2}$ and $L$ varies as $12,25,55 \mathrm{~nm}$ (solid blue, dashed red, and dot-dashed green, respectively).

The calculations above have been done assuming zero temperature $T$. If the superfluid phase exists at $T=0$, it may be also found at higher temperatures up to the critical temperature which can be found following Kosterlitz and Nelson [16]:

$$
T_{\mathrm{BKT}}=\frac{\pi \hbar^{2} n_{s}\left(T_{\mathrm{BKT}}\right)}{2 M}
$$

where $M$ is the exciton mass, $n_{s}$ is a superfluid density. As $n_{s}$ is a function of temperature, the above expression represents a transcendental equation for $T_{\mathrm{BKT}}$.

$n_{s}$ can be found as

$$
n_{s}=n-n_{n}\left(T_{\mathrm{BKT}}\right)
$$

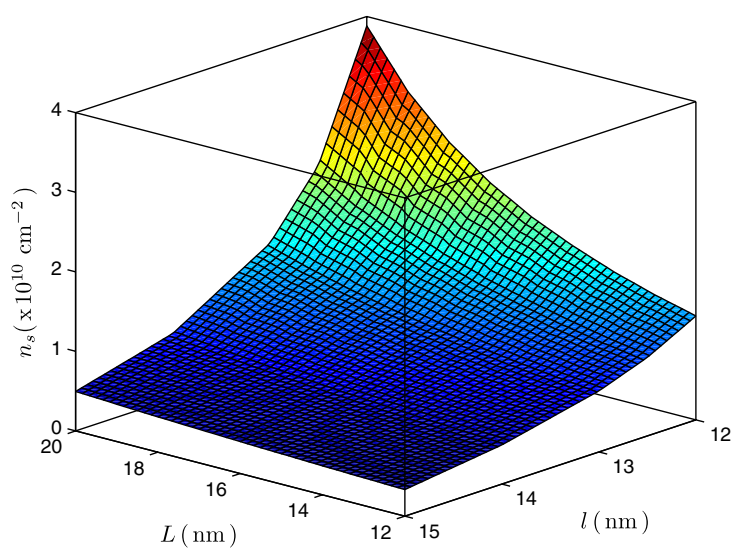

FIG. 3 (color online). Phase diagram of the system-the exciton BEC is unstable above the phase boundary surface, and stable below. Plotted for the same structure as in the previous figure. 


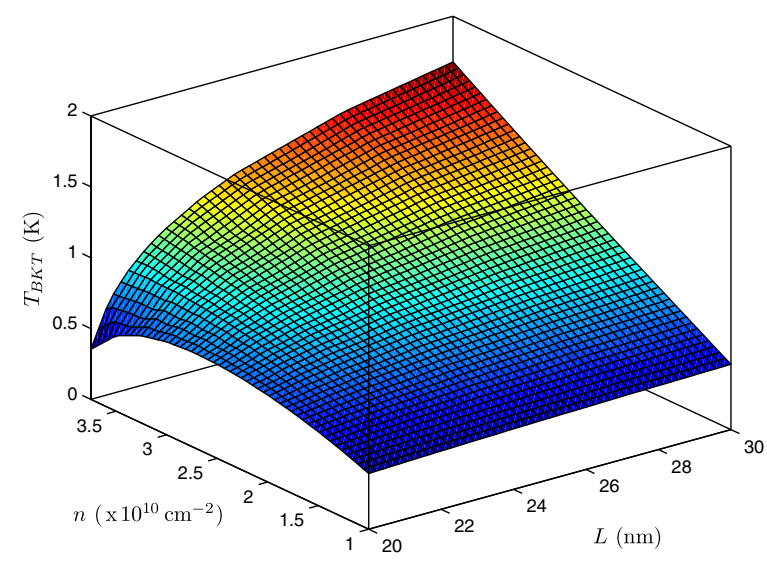

FIG. 4 (color online). Dependence of the BerezinskyKosterlitz-Thouless critical temperature on concentration and QW separation. Plotted for parameters as in previous figures.

where $n=N_{0} / A$ is a total $2 \mathrm{D}$ concentration of excitons (it equals $n_{s}$ at zero temperature), $n_{n}$ is a normal fraction concentration which can be estimated as in $[8,17,18]$,

$$
n_{n}(T, n)=\frac{\hbar^{2}}{2 \pi M k_{B} T} \int_{0}^{\infty} \frac{q^{3} e^{\hbar \omega(q) / k_{B} T}}{\left(e^{\hbar \omega(q) / k_{B} T}-1\right)^{2}} d q .
$$

The critical temperature $T_{\mathrm{BKT}}$ is influenced by the exciton-electron interaction as it depends on the modified dispersion of the collective modes (11). Figure 4 shows $T_{\mathrm{BKT}}$ for our system as a function of $L$ and $n$. One can see that at the large $L$ corresponding to a weak excitonelectron coupling, $T_{\mathrm{BKT}}$ is a linear function of the exciton concentration as should be expected for a conventional gas of interacting bosons. On the other hand, for small $L$ where the exciton-electron interaction is important, the critical temperature behaves nonmonotonously as a function of $n$ : it initially increases, then decreases at larger $n$. This manifests the gradual changes in the strength and sign of exciton-exciton interactions: while at low concentrations the excitons repel each other, at higher concentrations they start attracting each other at large $q$.

In conclusion, we have analyzed the spectrum of superfluid excitations in a hybrid exciton-electron system and found the roton minimum whose shape and depth depend on the exciton concentration and the spatial separation between excitons and electrons. The electron induced exciton-exciton attraction leads to a decrease of the BKT transition temperature and can eventually destroy the exciton BEC.

The authors thank H. Ouerdane and M. Portnoi for useful discussions. I. A. S. acknowledges support from RANNIS "Center of excellence in polaritonics." A.K. acknowledges the E.U. IRSES projects "Robocon" and "Polalas."

[1] M. Girardeau, J. Math. Phys. (N.Y.) 1, 516 (1960); Phys. Rev. 139, B500 (1965).

[2] M.T. Depue et al., Phys. Rev. Lett. 82, 2262 (1999).

[3] M. J. Bijlsma, B. A. Heringa, and H. T.C. Stoof, Phys. Rev. A 61, 053601 (2000).

[4] L. V. Butov, C. W. Lai, A. L. Ivanov, A. C. Gossard, and D. S. Chemla, Nature (London) 417, 47 (2002).

[5] L. V. Butov, J. Phys. Condens. Matter 19, 295202 (2007).

[6] F. P. Laussy, A. V. Kavokin, and I. A. Shelykh, Phys. Rev. Lett. 104, 106402 (2010).

[7] L. D. Landau, J. Phys. USSR 11, 91 (1947).

[8] A. Posazhennikova, Rev. Mod. Phys. 78, 1111 (2006).

[9] See, e.g., A. M. Zagoskin, Quantum Theory of Many-Body Systems (Springer, New York, 1998).

[10] H. Haug and S. W. Koch, Quantum Theory of the Optical and Electronic Properties of Semiconductors (World Scientific, Singapore, 1990).

[11] F. Tassone and Y. Yamamoto, Phys. Rev. B 59, 10830 (1999).

[12] C. Ciuti, V. Savona, C. Piermarocchi, A. Quattropani, and P. Schwendimann, Phys. Rev. B 58, 7926 (1998).

[13] A. L. Ivanov, Europhys. Lett. 59, 586 (2002).

[14] G. Ramon, R. Rapaport, A. Qarry, E. Cohen, A. Mann, Arza Ron, and L. N. Pfeiffer, Phys. Rev. B 65, 085323 (2002).

[15] H. Heiselberg, C. J. Pethick, H. Smith, and L. Viverit, Phys. Rev. Lett. 85, 2418 (2000).

[16] D. R. Nelson and J. M. Kosterlitz, Phys. Rev. Lett. 39, 1201 (1977).

[17] O. L. Berman, Yu. E. Lozovik, D. W. Snoke, and R. D. Coalson, Phys. Rev. B 70, 235310 (2004).

[18] G. Malpuech, D. D. Solnyshkov, H. Ouerdane, M. M. Glazov, and I. Shelykh, Phys. Rev. Lett. 98, 206402 (2007). 